Methods This study was conducted with 66 coronary heart disease (CHD) patients and 22 healthy adults. The blood specimens were collected before using Low molecular weight heparin (LMWH) inACS patients; and the blood specimens of Stable angina pectoris (SAP) patients and healthy adults were collected on the second morning with a fasting status. After centrifugal treatment, the plasma was saved in a Ultra-low temperature refrigerator. Coronary angiography was carried out on each one of the selected objects, and the quantity levels of total cholesterol (TC), triglyceride (TG), high density lipoprotein -cholesterol (HDL-C) and low density lipoprotein -cholesterol (LDL-C) was detected. The plasma TF and TFPI quantity was measured by ELISA.

Results The plasma TF antigen levels were highter in the Acute myocardial infarction (AMI) and Unstable angina pectoris (UAP) groups than in Stable angina pectoris (SAP) and control groups $(161.08 \pm 20.90$ and $152.76 \pm 20.66 \mathrm{pg} / \mathrm{ml}$ vs $99.72 \pm 16.75$ and $94.32 \pm 12.93 \mathrm{pg} / \mathrm{ml}, \mathrm{p}<0.05)$, there was no significant difference between the AMI group and UAP group, SAP group and control group ( $p>0.05)$. The plasma TFPI-1 antigen levels were highter in the AMI and UAP groups than in the SAP and control groups $(32.05 \pm 8.52$ and $31.49 \pm 10.61 \mathrm{ng} / \mathrm{ml}$ vs $19.93 \pm 9.22$ and $19.21 \pm 9.60 \mathrm{ng} / \mathrm{ml}, \mathrm{p}<0.05)$. The plasma TFPI-2 antigen levels were highter in the AMI and UAP groups than in the SAP and control groups $(4.56 \pm 0.96$ and $4.73 \pm 1.04 \mathrm{ng} / \mathrm{ml}$ vs $2.43 \pm 1.07$ and $2.06 \pm 0.64 \mathrm{ng} / \mathrm{ml}, \mathrm{p}<0.05)$.

Conclusions The plasma TF, TFPI-1, TFPI-2 antigen levels of ACS patients are higher than those of SAP patients and healthy adults, the result indicates ACS patients have an abnormally activity of coagulation system, the tissue factor pathway plays an important role in ACS patients, during our clinical work, we can reduce the incidence of coronary event through actively controlling plasma levels of tissue factor pathway in CHD patients; There were positive relationships between plasma TF, TFPI-1, TFPI-2 quantities and serum TC, LDL-C quantities, we can reduce the activity of tissue factor pathway through controlling the quantities of TC and LDL-C.

\section{e0449 EFFECTS OF CHOLESTEROL-LOWERING THERAPY WITH ROSUVASTATIN CALCIUM TABLET ON CORONARY FLOW RESERVE IN PATIENTS WITH UNSTABLE ANGINA PECTORIS}

doi:10.1136/hrt.2010.208967.449

Jiyan Zhang, Xiaodong Ge, Ping Xu, Suzhen Feng. Cardiology, People's Hospital of Shandong Province Cao County

Objective To investigate the effects of cholesterol-lowering therapy with rosuvastatin calcium tablet on coronary flow reserve (CFR) patients with unstable angina pectoris (UAP) and hypercholesterolaemia.

Method 40 patients with UAP were randomly divided into rosuvastatin calcium tablet therapeutic group $(n=20)$ and conventional therapeutic group $(n=20)$. By using colour Doppler ultrasound the coronary flow reserve was measured in patients with UAP before and after 3 rnonths cholesterol-lowering therapy.

Result After cholesterol-lowering therapy for 3 months the serum total cholesterol (TC), low density lipoprotein cholesterol (LDL-C), triglyceride (TG)decreased significaroly $\mathrm{p}<0.01$. The coronary flow, reserve was increased from $1.92 \pm 0.41$ to $2.97 \pm 0.62(p<0.01)$. An inverse correlation was found not only between TC and C FR $(r=-0.44, p<0.05)$ but also between LDL-C and CFR $(r=0.47$, $\mathrm{p}<0.05)$.

Conclusion Cholesterol-lowering therapy with rosuvastatin calcium tablet may improve coronary flow reserve in patients with UAP and hypercholesterolaemia.
Q0450 USEFULNESS OF THE NEUTROPHIL TO LYMPHOCYTE RATIO IN PREDICTING COMPLICATIONS IN PATIENTS WITH ACUTE MYOCARDIAL INFARCTION

doi:10.1136/hrt.2010.208967.450

Lu Yi, Fu Qiang, Liu Yi, Sheng Yang, Wei Peng. Department Of Cardiology, The Central Hospital Of Xuzhou, Xuzhou Jiangsu, China

Objective To investigate the relationship between the neutrophil to lymphocyte ratio and the morbidity of AMI complications.

Methods A total of 218 patients with ST segment elevated myocardial infarction whose blood routines were obtained at the admission were studied. All cases were divided into two groups according to the neutropil to lymphocyte ratio of small to large size. Then we analysed the morbidity of AMI complications between the two groups.

Results The peak value of cardiac troponin I was higher in group $\mathrm{B}((32.5 \pm 21.7) \mathrm{ng} / \mathrm{ml}$ and $(56.8 \pm 39.4) \mathrm{ng} / \mathrm{ml}$ respectively, $\mathrm{p}<0.01)$. The incidences of arrhythmia $(17.43 \%$ and $39.45 \%, p<0.01)$, heart failure $(22.94 \%$ and $51.38 \%, \mathrm{p}<0.01)$ and death $(2.75 \%$ and $11.01 \%$, $\mathrm{p}<0.05)$ were also higher in group $\mathrm{B}$. The multivariate stepwise regression analysis showed that the neutrophil to lymphocyte ratio and the morbidity of AMI complications had certain correlation.

Conclusions The neutrophil to lymphocyte ratio is a reliable indicator that can predict morbidity of AMI complications.

\section{Q0451 COMBINED USE OF OXIDISED LOW DENSITY LIPOPROTEIN AND C-REACTIVE PROTEIN FOR THE PREDICTION OF THE ACUTE CORONARY SYNDROME}

doi:10.1136/hrt.2010.208967.451

Zhang Ya-chen, Wei Jingjing, Chen Mantian, Zhou Qing, Meng Shu, Li Yigang. Division Of Cardiology, Xinhua Hospital, School Of Medicine, Shanghai Jiaotong University

Background Serum CRP levels can predict future risks among patients with stable and unstable angina, but CRP was easy infected by many factor. Increased blood levels of ox-LDL could play a role in these circumstances. However, Combined detection of ox-LDL and CRP for the prediction of the acute coronary syndrome are not known.

Methods All of the patients received a coronary angiography owing to complaining of chest pain. The coronary artery disease dianosis and stenosis severity was judged by two independent experts and the patients were accounting Gensini Score. The serum oxLDL and high sensitivity C-reactive protein (hs-CRP) levels were measured using a sandwich ELISA method. The MACEs of documented CAD patients were recorded in the one year follow-up period. SPSS software was chosen to analyse the influence of Oxidised Low Density Lipoprotein and C-reactive protein on the incidence of MACE.

Results (1) In acute myocardial infarction patients, ox-LDL and hsCRP levels were significantly higher than in patients with unstable angina pectoris $(p<0.01)$ or stable angina pectoris patients $(p<0.01)$ or in controls $(p<0.01)$ (acute myocardial infarction, oxLDL $177.5 \mathrm{mmol} / \mathrm{l}$, hs-CRP $21.4 \mathrm{mg} / \mathrm{l}$; unstable angina pectoris, oxLDL $97.5 \mathrm{mmol} / 1$, hs-CRP $6.7 \mathrm{mg} / 1$; stable angina pectoris, oxLDL $62.3 \mathrm{mmol} / \mathrm{l}$, hs-CRP $3.7 \mathrm{mg} / \mathrm{l}$; Control, oxLDL $41.7 \mathrm{mmol} / \mathrm{l}$, hs-CRP $2.7 \mathrm{mg} / \mathrm{l}$ ). (2) A positive correlation between the serum levels of oxLDL and CRP with the severity in patients with coronary artery disease. (3) Combined use of Oxidised Low Density Lipoprotein and C-reactive protein can predict the severity in patients with acute coronary syndrome and the risk for major adverse cardiac event (MACE) in patients with acute coronary syndrome $(\mathrm{p}<0.005)$.

Conclusions This study demonstrates that ox-LDL and hs-CRP levels show a significant positive correlation with the severity of 